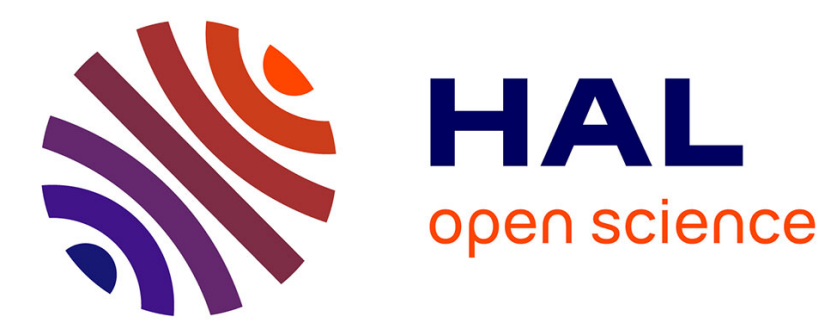

\title{
Clogging in constricted suspension flows
}

\author{
Alvaro Marin, Henri Lhuissier, Massimiliano Rossi, Christian Joachim Kähler
}

\section{To cite this version:}

Alvaro Marin, Henri Lhuissier, Massimiliano Rossi, Christian Joachim Kähler. Clogging in constricted suspension flows. Physical Review E , 2018, 97 (2), pp.21102. 10.1103/PhysRevE.97.021102 . hal01769088

\section{HAL Id: hal-01769088 \\ https://hal.science/hal-01769088}

Submitted on 17 Apr 2018

HAL is a multi-disciplinary open access archive for the deposit and dissemination of scientific research documents, whether they are published or not. The documents may come from teaching and research institutions in France or abroad, or from public or private research centers.
L'archive ouverte pluridisciplinaire HAL, est destinée au dépôt et à la diffusion de documents scientifiques de niveau recherche, publiés ou non, émanant des établissements d'enseignement et de recherche français ou étrangers, des laboratoires publics ou privés. 


\title{
Clogging in constricted suspension flows
}

\author{
Alvaro Marin, ${ }_{1}^{1}$ Henri Lhuissier, ${ }^{2}$ Massimiliano Rossi, ${ }^{3}$ and Christian J. Kähler ${ }^{3}$ \\ ${ }^{1}$ Physics of Fluids, University of Twente, The Netherlands \\ ${ }^{2}$ Aix-Marseille Université, CNRS, IUSTI, Marseille, France \\ ${ }^{3}$ Institut für Strömungsmechanik und Aerodynamik, Bundeswehr University Munich, Munich, Germany
}

(Received 29 May 2017; published 7 February 2018)

\begin{abstract}
The flow of a charged-stabilized suspension through a single constricted channel is studied experimentally by tracking the particles individually. Surprisingly, the behavior is found to be qualitatively similar to that of inertial dry granular systems: For small values of the neck-to-particle size ratio $(D / d<3)$, clogs form randomly as arches of the particle span the constriction. The statistics of the clogging events are Poissonian as reported for granular systems and agree for moderate particle volume fraction $(\phi \approx 20 \%)$ with a simple stochastic model for the number of particles at the neck. For larger neck sizes $(D / d>3)$, even at the largest $\phi(\approx 60 \%)$ achievable in the experiments, an uninterrupted particle flow is observed, which resembles that of an hourglass. This particularly small value of $D / d(\simeq 3)$ at the transition to a practically uninterrupted flow is attributed to the low effective friction between the particles, achieved by the particle's functionalization and lubrication.
\end{abstract}

DOI: 10.1103/PhysRevE.97.021102

Experience shows that rigid particles forced though a narrow constriction may either flow steadily, intermittently or do not flow at all when the particles form a clog obstructing the route [1]. Such flowing modes are encountered in different systems involving particle retention or discharge, such as the hopper of an emptying granular silo [2,3], the neck of an hourglass [4], microfluidic and filtration circuits where a loaded liquid enters a device or permeates a membrane [5-7], but also crowds of people evacuating a room or a hungry herd entering a room through a door $[1,8]$. However, besides their apparently similar behaviors, these systems conceal fundamentally different intermittency and clogging mechanisms. Hourglasses and silos contain dry granular media flowing collectively from a sedimented state to a gravity-driven flow limited by particles' interactions [2]. They clog when a stable static arch of particles spanning the constriction forms [9]. The clogging probability is essentially prescribed by the neck-toparticle size ratio $D / d$ and the particle shape [10] independently from the flow rate [11] and almost independently from the constriction angle [9] and other particle properties [10]. For ratios $D / d$ above typically $5-8$, both two-dimensional and three-dimensional flows are virtually uninterrupted $[9,10]$, although the critical status of this empirical assessment is still under debate [10,12-14].

By contrast, suspensions accommodate particle dilution and, for colloidal systems, often experience other clogging mechanisms involving single-particle effects mediated by wall-particle adhesion or particle aggregation [7,15,16]. At large concentrations, shear-induced particle migration becomes increasingly important $[17,18]$ and may, for instance, impede blood circulation in the smallest venules [19]. However, suspensions have also been reported to sustain clogging by arching, qualitatively similar to silos clogging [20-22], even at low volume fractions [23] and in the absence of adhesion or aggregation [24]. Although it is not always considered, this arching mechanism is crucial since it is expected to control clogging for suspensions of nonattractive particles. Moreover, the apparent similarity with silos is particularly surprising given the strong differences with a classical granular system: (1) The particle flow is driven by the viscous drag of the suspending liquid. (2) The particle concentration is variable. (3) Distant particle interactions, whether hydrodynamic or not, are present.

To determine the prevalence of clogging by arching in suspensions and the possible similarities with its dry counterparts we investigate the flow of a dilute suspension of charge-stabilized non-Brownian particles through a single microfluidic constriction with a controlled geometry. Using high-resolution and high-speed optical video microscopy, we track individual particles as they travel through the channel. This permits direct visualization of the clogging mechanism as well as obtaining the statistics of the clogging events by monitoring the precise number of particles that pass through the constriction before a clog forms.

The fluidic system consists of a straight channel of borosilicate glass (isotropic wet etching fabricated by Micronit microfluidics), which is locally constricted in the middle (see Fig. 1). The channel has a uniform thickness of $100 \mu \mathrm{m}$. The constriction is achieved by a linear narrowing of the channel with a half-angle of $45^{\circ}$ from the nominal width of $400 \mu \mathrm{m}$ down to $100 \mu \mathrm{m}$ at the neck. This forms a two-dimensional nozzle converging towards the almost square cross section of the neck $\left(110 \times 100 \mu \mathrm{m}^{2}\right.$-note that the flow itself is not two dimensional due to the boundary conditions). The suspensions consist of monodisperse polystyrene particles stabilized with negatively charged sulfate groups (Microparticles $\mathrm{GmbH}$ ) in a density-matched $21.5 \mathrm{wt} \%$ aqueous solution of glycerine (with a viscosity of $1.8 \mathrm{mPa}$ s). Different suspensions with particle diameters $d=98.5,59,48,41,33$, or $25 \mu \mathrm{m}( \pm 2 \%)$ are used. Adopting the neck width $D=100 \mu \mathrm{m}$ as the characteristic length scale, these correspond to neck-to-particle ratios $D / d=1.02,1.7,2.07,2.43,3.03$, and 4 , respectively. The 


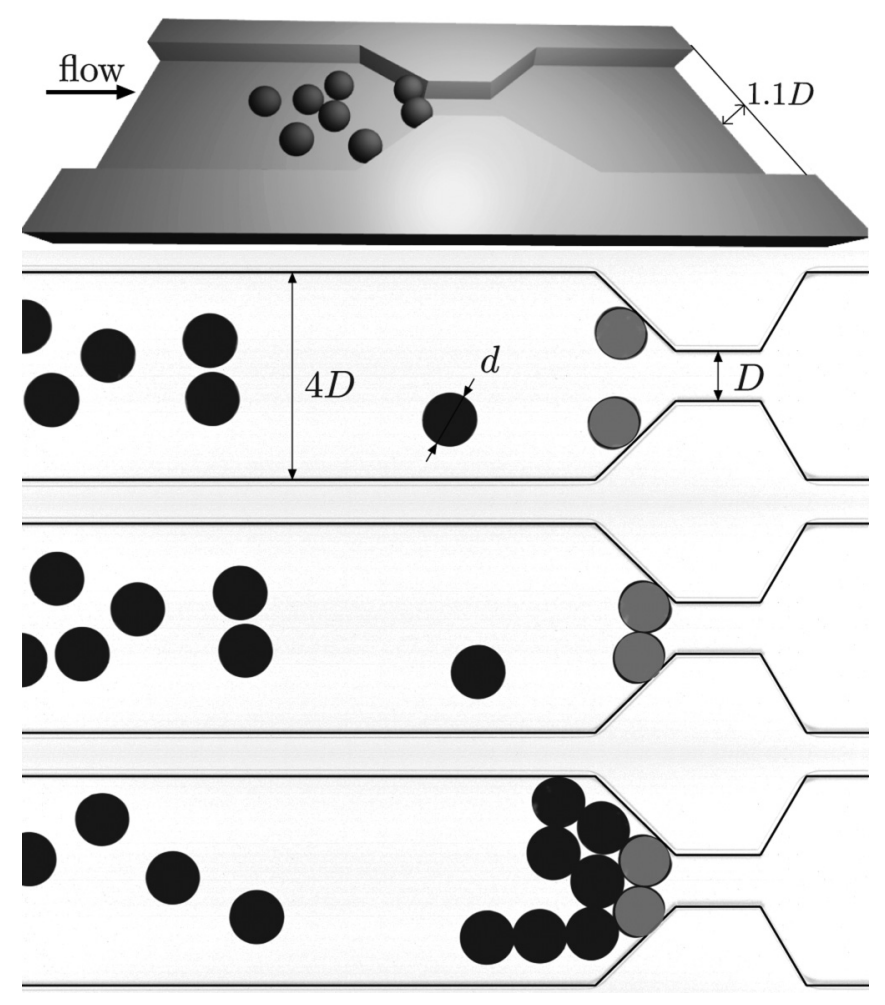

FIG. 1. Top: Schematics of the system; a laterally constricted channel with uniform thickness. A suspension of particles with a diameter $d$ smaller than the neck width $D$ and nominal particle volume fraction $\phi$ is forced through the constriction until a clog forms. Bottom: Image sequence showing the formation of a clog for $D / d=1.02$ when a sufficient number of particles (here two) reach the constriction at the same time.

charged sulfate groups confer them a small negative surface potential (on the order of $-50 \mathrm{mV}$ ) but sufficient to prevent both their agglomeration and their adhesion to the channel walls (borosilicate glass). The suspensions are prepared with a particle volume fraction of about $2 \%$, preconcentrated up to approximately $20 \%$ and inserted in the device. However, the homogeneity of the volume fraction in the channel cannot be controlled precisely, mainly due to the successive clogging and flow reversals needed to acquire the statistics (see below). We therefore measure the particle volume fraction $\phi$ in situ, close to the constriction, by directly imaging and counting the particles. This yields $\phi=20 \pm 10 \%$ for all $D / d$ smaller than 3 where the \pm refers to slow variations in time (the time-averaged particle volume fraction was found to be uniform in the channel). For the largest particle-to-neck ratios $(D / d>3), \phi$ was eventually increased up to $\approx 60 \%$ in order to promote clogging events (see below). The suspensions are driven through the constriction by a pressure-based flow controller (Fluigent) at a Reynolds number smaller than $10^{-2}$ (corresponding to particle velocities of $\gtrsim 1 \mathrm{~mm} / \mathrm{s}$ ). Identical results have been obtained with a volume-driven flow using a syringe pump. Note that most of the data have been obtained with dilute suspensions $(\phi \approx 20 \%)$ for which the particle flow rate is set only by the liquid flow rate and the particle volume fraction. For each neck-to-particle ratio $D / d$, experiments have been performed sequentially in a semiautomatic routine. After a clogging event, the direction of the flow is reversed. The constriction unclogs immediately, and the flow is maintained in the reverse direction for a few seconds. As the reversed flow is stopped, the camera is activated, and the flow in the regular direction is restored. When a clogging event is detected, the camera is stopped, and the routine is repeated. The routine has been repeated at least 100 times for each neck-to-particle ratio. Successive clogging events are not affected by each other, see the Supplemental Material [25] for Fig. S1 in which the number of escapees of each event is plotted versus that of the previous event. The lack of correlation between successive events is illustrated by the random spreading of the data.

The experiment starts when the flow is switched on and particles are dragged by the flow towards the constriction. We count the number of particles passing through the constriction until the channel clogs, which we note as $s$ and refer as the number of escapees. Due to the low adherence of the particles to the walls and to each other, the arch is fully disassembled by reversing the flow for a short time. The forward flow is then restored, and a new independent clogging event is observed (the independency was verified from the absence of correlation between successive clogging events, see the Supplemental Material [25] for Fig. S1). For each of them, approximately 100 clogging events are analyzed. A typical histogram of $s$ is shown in Fig. 2. The number $N$ of events observed for a given number of escaped particles clearly decreases with increasing $s$, and the experimental probability $N(s) / \sum_{s^{\prime}=0}^{\infty} N\left(s^{\prime}\right)$ is well fitted by an exponential distribution,

$$
p(s)=\frac{1}{\langle s\rangle} e^{-s /\langle s\rangle},
$$

which suggests that the clogging events can be described as a random Poisson process as already observed for dry granular systems $[10,12]$. This assessment is also supported by analyzing the standard deviation of the number of escaped particles $\sqrt{\left\langle s^{2}\right\rangle}$ shown in the inset of Fig. 2. which is found to be close to the value of $\sqrt{2}\langle s\rangle$ expected for an exponential distribution. Such behavior has been found for all experiments with $D / d<3$ in which clogging events could be observed.

The data for $D / d<3$ are summarized in Fig. 2 in terms of the cumulative probability $P(s /\langle s\rangle)=\sum_{s^{\prime}=0}^{s} p\left(s^{\prime}\right)$, i.e., the probability of having less than $s$ particles passing through the constriction. As shown in Fig. 2, the probabilities, normalized by the average number of escapees $\langle s\rangle$, collapse for all the data sets. This suggests that particles, or small groups of particles, pass through the constriction independent from each other and have all the same probability of clogging the constriction, regardless of the history of clogging events. Note that the physical reason underpinning this independency is not the same as for dry granular flows. Whereas for granular media it results from the saturation of the lithostatic pressure at the scale of the silo aperture [4], it is, presently, a mere consequence of the moderate particle volume fraction of the suspension. Despite this difference, the statistical analogy is strong, and the clogging statistics are entirely given by $\langle s\rangle$.

The particle flow rate and and the particle volume fraction fluctuate in our experiments within a limited range. Despite the fluctuations, the mean number of escapees is not significantly affected. In order to confirm this, two additional sets of 

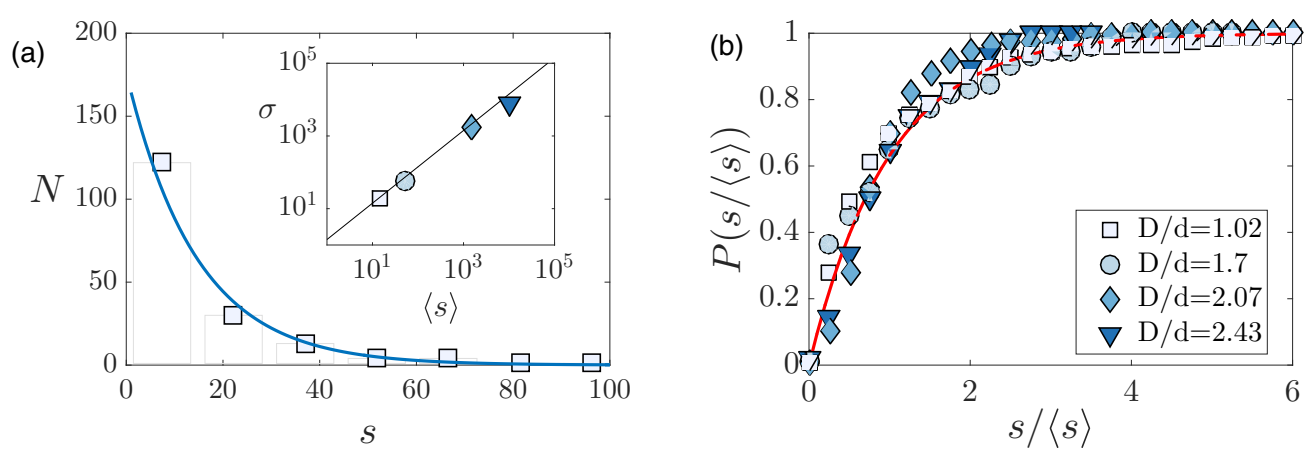

FIG. 2. (a) Histogram of the number of escaped particles $s$ for $D / d=1.02$. The number of events $N$ decays with increasing $s$. The line represents the exponential distribution $\sum_{s^{\prime}=0}^{\infty} N\left(s^{\prime}\right)\langle s\rangle^{-1} e^{-s /\langle s\rangle}$, having the same mean value of $\langle s\rangle=14.5$ as the data. The inset: Standard deviation of the number of escapees $\sqrt{\left\langle s^{2}\right\rangle}$ compared to the value of $\sqrt{2}\langle s\rangle$ (the solid line) expected for an exponential distribution for $D / d=1.02(\boldsymbol{\nabla}), 1.7(\bullet), 2.07(\checkmark), 2.43(\nabla)$. (b) Cumulative clogging probability of the relative number of escapees $s /\langle s\rangle$ for neck-to-particle size ratios covering a $10^{3}$-fold range of a mean number of escapees $\langle s\rangle$ (see Fig. 5). The solid line is the curve $P=1-e^{-s /\langle s\rangle}$ expected for an exponential distribution.

experiments have been conducted in which the average particle flow rate and the average particle volume fraction, respectively, were varied. In these sets of experiments, the particle volume fraction was varied by changing the way the particles were loaded, whereas the velocity was varied by changing the driving pressure at the inlet. For the typical flow rate considered here $(\sim 1 \mathrm{~mL} / \mathrm{h}),\langle s\rangle$ is found to be practically independent of the flow rate but strongly dependent on $D / d$. See the Supplemental Material [25] for Fig. S2 where the number of escapees per experiment is plotted in the range of velocities and volume fractions accessible experimentally.

Figure 3 presents a sample of raw data for the case of $D / d=2.07$. The left panel shows the instantaneous number of particles in the channel portion immediately upstream of the constriction. The central panel shows $\phi(t)$, the time-dependent volume fraction averaged over this portion. Typically, the number of particles (volume fraction) reaches a plateau after a short transient. Although fluctuations persist until the constriction clogs, we should remark that there is no correlation between the maxima in the number of particles and the clogging events. Finally, the right panel shows the (cumulated) number of escaped particles as a function of time, which stops increasing once a clog event (indicated by a vertical line) occurs.

We therefore turn to the mean number of escapees $\langle s\rangle$. Not surprisingly, $\langle s\rangle$ increases, i.e., clogging events become less likely, as the particles become smaller. For $D / d=1.02$ [Fig. 4(a)], a clogging event typically occurs after only $\langle s\rangle \approx$
15 particles have passed through the aperture. By doubling $D / d$ to 2.07 [Fig. 4(b)], the average number of escapees $\langle s\rangle$ rises to as much as $\approx 1500$. As Fig. 5 shows, this steep increase with $D / d$ perseveres for larger values of the neck-to-particle size ratio. Actually, for $D / d=4$ the probability of clogging is so small that no permanent clogs could be observed-hence no statistics of $s$ could be obtained. More surprisingly, even after increasing $\phi$ up to the largest particle volume fraction $\phi \approx 60 \%$ achievable in our system, the flow could be maintained over several hours at a volume flow rate of $\sim 1 \mathrm{~mL} / \mathrm{h}$ without any interruption. That is to say, no persistent clog had formed after typically $10^{8}$ particles had passed through the constriction (implying $\langle s\rangle \gtrsim 10^{8}$ ). For most practical purposes such a flow can probably be considered as uninterrupted, which should be all the more true for lower $\phi$ since $\langle s\rangle$ is expected to be even larger. It is however not possible to conclude on whether the clogging probability has identically vanished or if it has only dramatically decreased to a value smaller than $10^{-8}$ despite $\phi \approx 60 \%$. Although no permanent clog is observed for $D / d>3$, different flows develop at large $\phi$ 's. For $D / d=3.03$ the flow is intermittent, i.e., particle flow occurs in erratic bursts, separated by short periods of arrest (of a few seconds). During each particle burst, approximately $10^{4}-10^{5}$ particles can typically escape. Whereas for $D / d>4$ the flow is continuous [Fig. 4(c)] as already observed with dry grains (hourglass) [4] and colloidal suspensions [26].
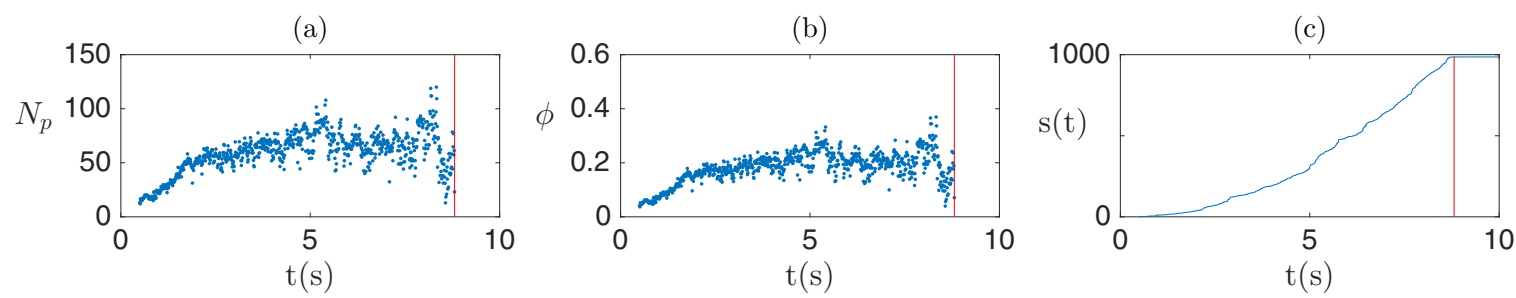

FIG. 3. Sample of raw data of an individual measurement for the case of $D / d=2.07$. (a) Instantaneous number of particles in the channel portion immediately upstream from the constriction (having an approximate length of $700 \mu \mathrm{m}$ ). (b) Time-dependent volume fraction averaged over this portion. (c) Cumulative number of escapees. The red vertical line indicates the clog occurrence (note that, subsequently, the number of escapees remains constant). 
(a)

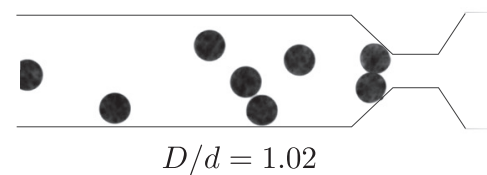

(b)

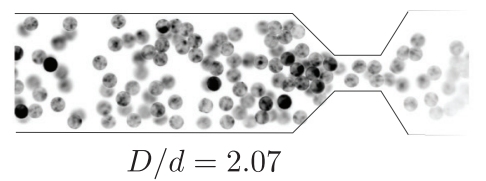

(b)

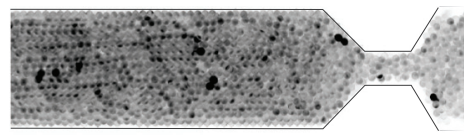

$D / d=4$

FIG. 4. Snapshots of the flow at the constriction. Images (a) and (b) show the configuration immediately after a clog has formed for $D / d=1.02$ and $D / d=2.07$. Panel (c) shows the continuous and uninterrupted flow observed for $D / d=4$ : even at the largest particle volume fraction $(\phi \approx 60 \%)$, no clog is observed (i.e., after $\sim 10^{8}$ particles through the constriction).

In order to explain the clogging behavior of the constriction, we now proceed to build a simple statistical model describing the average number of escapees $\langle s\rangle$ for low particle volume fractions. We base our model on two observations. First, in the present case of nonadhesive particles, the particles do not aggregate and remain almost randomly dispersed in the suspending liquid. Second, arches do not build up progressively from the accumulation of successive particles [15] but they form suddenly. As shown in Fig. 1, a stable arch is formed when a sufficient number of particles $n$ reach the constriction within a sufficiently short time interval, that is to say, when $n$ particles are contained at the same time in a given critical volume $\Omega$ prescribed by the geometry of the constriction and the particles' properties. Now, the probability that, among particles with a nominal number density of $c \equiv 6 \phi / \pi d^{3}$ and animated by random and independent motions, exactly $n$ particles lie at the same time in a given arbitrary volume $\Omega$ is

$$
q=\frac{(\Omega c)^{n-1}}{(n-1) !} e^{-\Omega c}
$$

This probability strongly decreases with increasing values of $n$. The probability of forming a clog is therefore expected to be dominated by the minimal number of particles required to form an arch, i.e, by

$$
n \simeq \frac{D^{2}}{d^{2}}+1,
$$

assuming the minimal stable arch typically spans the neck's cross section. The critical volume $\Omega$ in which these particles must lie to form a clog on their arrival at the constriction can be

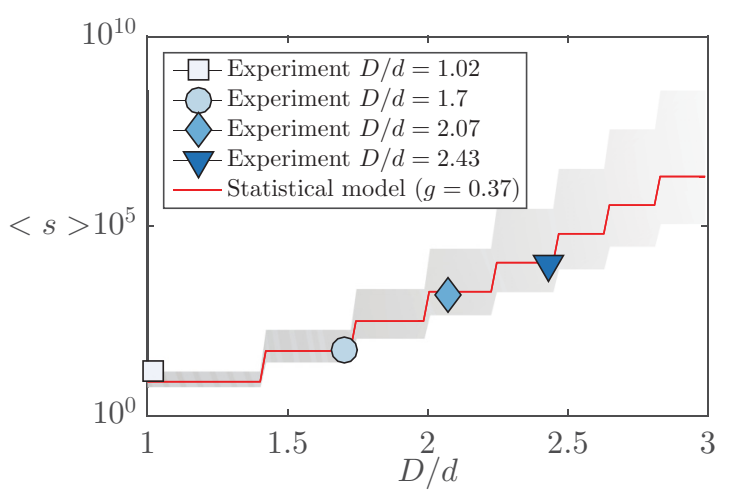

FIG. 5. Average number of escapees $\langle s\rangle$ observed experimentally and predicted by the stochastic model Eq. (6) with $g(\mu)=0.37$. The solid line refers to the prediction for the mean particle volume fraction $\phi=20 \%$. The gray band represents deviations from the predicted $\langle s\rangle$ value due to unintended changes in volume fraction $10 \%<\phi<30 \%$. thought of as the typical volume span by the particles centered in all possible conformations corresponding to a stable arch (see Ref. [9]). $\Omega$ is expected to depend upon $n$ as well as upon the particle interactions and the angle of the constriction. We assume that it is essentially set by the volume of the $n$ particles modulated by an effective friction coefficient $\mu$ through a geometrical prefactor $g(\mu)$ according to

$$
\Omega=g(\mu) \frac{n \pi d^{3}}{6} .
$$

In the limit $s \gg n$, the probability $p(s)$ that a clog forms after the escape of exactly $s$ particles simply verifies $\partial_{s}(p / q)=$ $-p$. Making use of Eqs. (1) and (4) and considering a steady nominal volume fraction (constant $\phi$ ), this yields the following distribution and average number of escapees:

$$
\begin{gathered}
p(s)=q e^{-q s}, \\
\langle s\rangle=q^{-1}=\frac{(n-1) !}{(g \phi n)^{n-1}} e^{g \phi n} .
\end{gathered}
$$

Together with Eq. (3), this makes the neck-to-particle ratio $D / d$ explicit.

For a given $D / d$, Eq. (6) recovers the experimental exponential distribution of $s$ given in Eq. (1). It also predicts a steep increase in the average number of escapees as a function of the neck-to-particle ratio since $\langle s\rangle \sim(D / d)\left(g \phi e^{1-g \phi}\right)^{-(D / d)^{2}}$. Equation (6) is compared to the experimental statistics in Fig. 5 for all values of $D / d$ for which clogs have been observed $(D / d<3)$. Considering the nominal particle volume fraction $\phi=0.2 \pm 0.1$, a single parameter remains, namely, the geometrical function $g$ appearing in $\Omega$. Taking $g(\mu)=$ 0.37 , and besides its crude assumptions, the model predicts surprisingly well the experimental results over the whole range of neck-to-particle size ratios.

In order to test the influence of particle repulsion on the clogging probability, a set of experiments has also been performed with the largest particles $(D / d=1.02)$ and $1 \mathrm{~mol} / \mathrm{l}$ of sodium chloride added to the suspending liquid. An increase in the free charge density in the solution reduces the thickness of the repulsive Debye layer, which helps maintain a liquid layer between the particles. The addition of salt is therefore expected to facilitate solid contacts which, in turn, should increase the effective particle-particle and particle-wall friction during clog formation. We found that the addition of salt reduced the number of escapees to $\langle s\rangle=9.4$ as compared to $\langle s\rangle=14.5$ without salt. This is consistent with Eqs. (4)-(6) since a larger effective friction between particles is expected to increase the envelope of the stable arch structure, i.e., the critical volume $\Omega$ in which the particles must lie, therefore 
reducing $\langle s\rangle$. We must add that a few particle clusters were also observed in the flow when salt was added. Whether these particles were irreversibly aggregated or not was not possible to determine.

To conclude, we have shown that nonadhesive colloidal particles in suspension flow across constrictions in a strikingly similar way as dry noncohesive granular systems do. At low Reynolds numbers and in the absence of particle aggregation or particle-wall adhesion, the neck-to-particle size ratio $D / d$ determines the flow regime (clogged, intermittent or continuous), independently from the flow rate. At low $D / d$, the flow is interrupted by the formation of stable particle arches spanning the constriction. The clogging events follow Poisson statistics which can be described in the diluted regime with a simple stochastic model for the number of particles forming the arch. Interestingly, this stochasticity has a different origin as for dry granular flows. Whereas for the latter the loss of memory is a consequence of the multiple particle collisions, in the present dilute case it simply stems from the random arrival of the particles at the neck. At larger $D / d>3$ 's, the flow becomes uninterrupted over the longest experimental duration achiev- able ( $\sim 10^{8}$ particles), even upon dramatically increasing the particle volume fraction to $\phi \approx 60 \%$. This apparently continuous flow regime appears for lower values of $D / d$ than for dry granular systems $[9,10]$. This is consistent with the decrease in the critical $D / d$ with decreasing the particle-particle friction coefficient reported for dry systems $[9,12]$ if one assumes that the lubrication forces and the repulsive dielectric forces maintain a liquid layer between the particles, which results in a low effective friction [27]. This suggests that, even in the absence of adhesion, the flow of particles through confined geometries can be significantly facilitated by engineering short-range repulsive forces (of electrostatic or polymer- or surfactantinduced nature) and smoother surfaces. Such a feature could be exploited to enhance the functional range of fluidic devices, filters, and membranes intended for suspensions.

The authors acknowledge several useful and always stimulating discussions with I. Zuriguel and the technical support of A. Volk in the experiments. The project has been financially supported by the Deutsche Forschungsgemeinschaft (DFG) Grant No. KA1808/12.
[1] I. Zuriguel, D. R. Parisi, R. C. Hidalgo, C. Lozano, A. Janda, P. A. Gago, J. P. Peralta, L. M. Ferrer, L. A. Pugnaloni, É. Clément et al., Sci. Rep. 4, 7324 (2014).

[2] W. A. Beverloo, H. A. Leniger, and J. Van de Velde, Chem. Eng. Sci. 15, 260 (1961).

[3] S. D. Kulkarni, B. Metzger, and J. F. Morris, Phys. Rev. E 82, 010402 (2010).

[4] X.-1. Wu, K. J. Maloy, A. Hansen, M. Ammi, and D. Bideau, Phys. Rev. Lett. 71, 1363 (1993).

[5] K. V. Sharp and R. J. Adrian, Microfluid. Nanofluid. 1, 376 (2005).

[6] J. Lin, D. Bourrier, M. Dilhan, and P. Duru, Phys. Fluids 21, 073301 (2009).

[7] P. Bacchin, A. Marty, P. Duru, M. Meireles, and P. Aimar, Adv. Colloid Interface Sci. 164, 2 (2011).

[8] D. Helbing, I. Farkas, and T. Vicsek, Nature (London) 407, 487 (2000).

[9] K. To, P.-Y. Lai, and H. K. Pak, Phys. Rev. Lett. 86, 71 (2001).

[10] I. Zuriguel, A. Garcimartín, D. Maza, L. A. Pugnaloni, and J. M. Pastor, Phys. Rev. E 71, 051303 (2005).

[11] S. Dorbolo, L. Maquet, M. Brandenbourger, F. Ludewig, G. Lumay, H. Caps, N. Vandewalle, S. Rondia, M. Mélard, J. van Loon et al., Granular Matter 15, 263 (2013).

[12] K. To, Phys. Rev. E 71, 060301 (2005).

[13] T. Masuda, K. Nishinari, and A. Schadschneider, Phys. Rev. Lett. 112, 138701 (2014).
[14] C. C. Thomas and D. J. Durian, Phys. Rev. Lett. 114, 178001 (2015).

[15] H. M. Wyss, D. L. Blair, J. F. Morris, H. A. Stone, and D. A. Weitz, Phys. Rev. E 74, 061402 (2006).

[16] G. Agbangla, P. Bacchin, and E. Climent, Soft Matter 10, 6303 (2014).

[17] A. Karnis, H. L. Goldsmith, and S. G. Mason, J. Colloid Interface Sci. 22, 531 (1966).

[18] T. Moraczewski and N. C. Shapley, Phys. Fluids 18, 123303 (2006).

[19] R. Zhou and H.-C. Chang, J. Colloid Interface Sci. 287, 647 (2005).

[20] A. Guariguata, M. A. Pascall, M. W. Gilmer, A. K. Sum, E. D. Sloan, C. A. Koh, and D. T. Wu, Phys. Rev. E 86, 061311 (2012).

[21] P. G. Lafond, M. W. Gilmer, C. A. Koh, E. D. Sloan, D. T. Wu, and A. K. Sum, Phys. Rev. E 87, 042204 (2013).

[22] J. Koivisto and D. J. Durian, Phys. Rev. E 95, 032904 (2017).

[23] V. Ramachandran and H. S. Fogler, J. Fluid Mech. 385, 129 (1999).

[24] N. Roussel, T. L. H. Nguyen, and P. Coussot, Phys. Rev. Lett. 98, 114502 (2007).

[25] See Supplemental Material at http://link.aps.org/supplemental/ 10.1103/PhysRevE.97.021102 for additional technical information and a video of a sample experiment.

[26] D. Genovese and J. Sprakel, Soft Matter 7, 3889 (2011).

[27] C. Clavaud, A. Bérut, B. Metzger, and Y. Forterre, Proc. Natl. Acad. Sci. U.S.A. 114, 5147 (2017). 